\title{
MHD Two-Fluid Flow and Heat Transfer between Two Inclined Parallel Plates in a Rotating System
}

\author{
P. Sri Ramachandra Murty and G. Balaji Prakash \\ Department of Mathematics, GIT, GITAM University, Visakhapatnam 530 045, India \\ Correspondence should be addressed to P. Sri Ramachandra Murty; drmurtypsr@yahoo.com
}

Received 17 April 2014; Revised 18 November 2014; Accepted 23 November 2014; Published 17 December 2014

Academic Editor: Majid Ghassemi

Copyright (c) 2014 P. Sri Ramachandra Murty and G. Balaji Prakash. This is an open access article distributed under the Creative Commons Attribution License, which permits unrestricted use, distribution, and reproduction in any medium, provided the original work is properly cited.

\begin{abstract}
Two-phase magnetohydrodynamic convective flow of electrically conducting fluid through an inclined channel is studied under the action of a constant transverse magnetic field in a rotating system. The fluids in the two phases are steady, incompressible, laminar, immiscible, and electrically conducting, having different densities, viscosities, and thermal and electrical conductivities. The transport properties of both the fluids are assumed constant. The bounding infinite inclined parallel plates are maintained at different constant temperatures, making an angle $\phi$ with the horizontal. Approximate solutions for velocity and temperature distributions are obtained by using a straightforward regular perturbation technique. An in-depth study has been done on the effects of rotation parameter, Hartmann number, inclination angle, the ratio of electrical conductivities, and viscosities of two fluids on the flow. It is observed that the effect of increasing rotation is to decrease the primary velocity. Further it is noticed that as the rotation increases, the secondary velocity increases for smaller rotation, while for larger rotation it decreases. It is also found that the temperature distribution decreases as the rotation increases.
\end{abstract}

\section{Introduction}

The study of convective Hartmann flow and heat transfer between two parallel plates is receiving considerable interest in the current literature. Shail [1] studied the problem of Hartmann flow of a conducting fluid in a horizontal channel of insulated plates with a layer of nonconducting fluid overlying a conducting fluid in a two-fluid flow. Rudraiah et al. [2] and Umavathi [3] have done a detailed analysis on free and forced convective heat transfer to an electrically conducting fluid in a channel. Lohrasbi and Sahai [4], Malashetty and Leela $[5,6]$, and Raju and Murty [7] have studied the Hartmann flow characteristics of two fluids in a horizontal channel. Seth et al. [8] have presented the detailed analysis on Hartmann flow in a rotating system in the presence of inclined magnetic field with Hall effects. Chauhan and Rastogi $[9,10]$ have considered Hall current and heat transfer effects on MHD flow and MHD Couette flow in a channel partially filled with a porous medium in a rotating system.

Numerous publications dealing with both the experimental and the theoretical aspects of the two-phase flow systems with or without considering the heat transfer problems associated with MHD power generators, MHD devices, and thermonuclear power generations have appeared in the literature. Abdul Mateen [11, 12] has studied the magnetohydrodynamic flow and transient magnetohydrodynamic flow of two immiscible fluids through a horizontal channel. Raju and Nagavalli [13] have studied the MHD two-layered unsteady flow and heat transfer through a horizontal channel in the presence of applied magnetic and electric fields in a rotating system. Recently, Murty and Linga Raju [14] have investigated magnetohydrodynamic two-phase flow and heat transfer between two parallel porous walls in a rotating system.

Basically, the inclined geometry has enormous applications in the heat transfer technology like solar collector. Though such problems with inclined geometry are close to realistic practical situations, much attention has not been given to handling them except the studies by Malashetty and Umavathi [15], Malashetty et al. [16], S. Daniel and Y. S. Daniel [17], and Murty and Prakash [18]. Therefore, in this paper, we have studied two-phase magnetohydrodynamic 
convective flow of electrically conducting fluid through an inclined channel under the action of a constant transverse magnetic field when rotated by an angular velocity about an axis perpendicular to the plates.

\section{Mathematical Formulation}

A steady laminar and fully developed two-phase magnetohydrodynamic convective flow driven by a common constant pressure gradient $(-\partial p / \partial x)$ and temperature gradient $\Delta T=$ $\left(T_{w 1}-T_{w 2}\right)$ has been considered in the presence of a constant magnetic field applied transversely to the direction of the flow. The physical configuration is shown in Figure 1, which consists of two infinite inclined parallel plates maintained at different constant temperatures, extending in the $x$ and $z$ directions, making an angle $\phi$ with the horizontal. The regions $0 \leq y \leq h_{1}$ and $-h_{2} \leq y \leq 0$ are occupied by two different electrically conducting incompressible fluids having density $\rho_{i}$, viscosity $\mu_{i}$, electrical conductivity $\sigma_{i}$, and thermal conductivity $K_{i}$. The whole system is rotated with an angular velocity $\Omega$ in a counterclockwise direction about $y$ axis perpendicular to the plates. The transport properties of both the fluids are assumed constant. The suffix $i(i=1,2)$ represents the values for phases I and II, respectively.

With these assumptions, the governing equations of motion and energy for Boussinesq fluids as in Malashetty et al. [16] for both phases are

$$
\begin{gathered}
\mu_{i}\left(\frac{d^{2} u_{i}}{d y^{2}}\right)+\rho_{i} g \beta_{i}\left(T_{i}-T_{w 2}\right) \sin \phi-\sigma_{i} B_{0}^{2} u_{i} \\
=\left(\frac{\partial p}{\partial x}\right)+2 \Omega \rho_{i} w_{i} \\
\mu_{i}\left(\frac{d^{2} w_{i}}{d y^{2}}\right)-\sigma_{i} B_{0}^{2} w_{i}=-2 \Omega \rho_{i} u_{i}, \\
\left(\frac{d^{2} T_{i}}{d y^{2}}\right)+\left(\frac{\mu_{i}}{K_{i}}\right)\left\{\left(\frac{d u_{i}}{d y}\right)^{2}+\left(\frac{d w_{i}}{d y}\right)^{2}\right\} \\
+\left[\frac{\sigma_{i} B_{0}^{2}\left(u_{i}^{2}+w_{i}^{2}\right)}{K_{i}}\right]=0,
\end{gathered}
$$

where $u_{i}$ and $w_{i}$ are the primary and secondary velocity components along $x$ and $z$ directions, respectively, $T_{i}$ is the temperature, $\beta_{i}$ is the coefficient of thermal expansion, and $g$ is the acceleration due to gravity. The fluid and the thermometric boundary conditions are unchanged by the addition of electromagnetic field. The no-slip condition requires that the velocity must be vanishing at the wall. In addition, the fluid velocity, shear stress, temperature, and heat flux must be continuous across the interface.

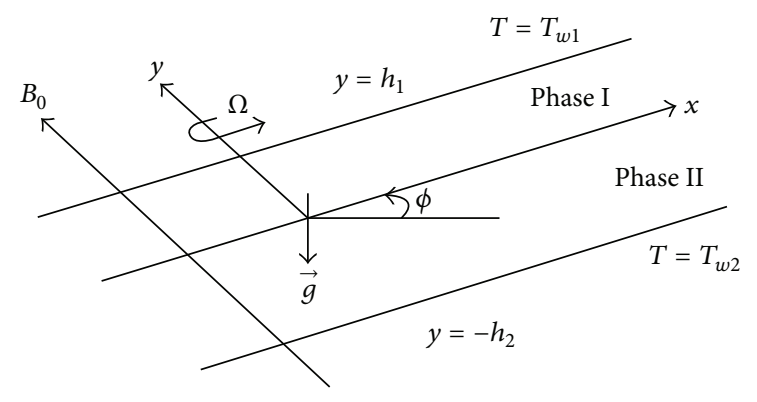

FIgURE 1: Physical configuration.

The boundary and interface conditions are

$$
\begin{gathered}
u_{1}\left(h_{1}\right)=0, \\
w_{1}\left(h_{1}\right)=0 ; \\
u_{1}(0)=u_{2}(0), \\
w_{1}(0)=w_{2}(0) \\
u_{2}\left(-h_{2}\right)=0 \\
w_{2}\left(-h_{2}\right)=0 \\
\mu_{1}\left(\frac{d u_{1}}{d y}\right)=\mu_{2}\left(\frac{d u_{2}}{d y}\right), \\
\mu_{1}\left(\frac{d w_{1}}{d y}\right)=\mu_{2}\left(\frac{d w_{2}}{d y}\right), \\
T_{1}\left(h_{1}\right)=T_{w 1}, \\
T_{1}(0)=T_{2}(0), \\
T_{2}\left(-h_{2}\right)=T_{w 2}, \\
K_{1}\left(\frac{d T_{1}}{d y}\right)=K_{2}\left(\frac{d T_{2}}{d y}\right) \quad \text { at } y=0 .
\end{gathered}
$$

In making these equations dimensionless, the following transformations are used:

$$
\begin{aligned}
u_{i}^{*} & =\frac{u_{i}}{\bar{u}_{1}}, \\
y_{i}^{*} & =\frac{y_{i}}{h_{i}}, \\
\theta & =\frac{\left(T-T_{w 2}\right)}{\Delta T}, \\
m & =\frac{\mu_{1}}{\mu_{2}}, \\
K & =\frac{K_{1}}{K_{2}},
\end{aligned}
$$




$$
\begin{aligned}
& h=\frac{h_{2}}{h_{1}}, \\
& n=\frac{\rho_{2}}{\rho_{1}} \text {, } \\
& b=\frac{\beta_{2}}{\beta_{1}}, \\
& s=\frac{\sigma_{2}}{\sigma_{1}}, \\
& \mathrm{Gr}=\frac{\left(g \beta_{1} h_{1}^{3} \Delta T\right)}{v_{1}^{2}}, \\
& M=B_{0} h_{1} \sqrt{\frac{\sigma_{1}}{\mu_{1}}}, \\
& \operatorname{Pr}=\frac{\mu_{1} C_{p}}{K_{1}} \text {, } \\
& \mathrm{Ec}=\frac{\bar{u}_{1}^{2}}{C_{p} \Delta T}, \\
& \operatorname{Re}=\frac{\left(\bar{u}_{1} h_{1}\right)}{v_{1}}, \\
& P=\left(\frac{h_{1}^{2}}{\mu_{1} \bar{u}_{1}}\right)\left(\frac{\partial p}{\partial x}\right) \text {, } \\
& R^{2}=\frac{\Omega h_{1}^{2}}{v} .
\end{aligned}
$$

With the above nondimensional quantities, the governing equations (1) become

$$
\begin{aligned}
& \left(\frac{d^{2} u_{i}}{d y^{2}}\right)+\left(\frac{\mathrm{Gr}}{\mathrm{Re}}\right) A \sin \phi \theta_{i}-B M^{2} u_{i}=\mathrm{CP}+2 R^{2} w_{i}, \\
& \left(\frac{d^{2} w_{i}}{d y^{2}}\right)-B M^{2} w_{i}=-2 R^{2} u_{i}, \\
& \left(\frac{d^{2} \theta_{i}}{d y^{2}}\right)+\operatorname{PrEc} D\left[\left(\frac{d u_{i}}{d y}\right)^{2}+\left(\frac{d w_{i}}{d y}\right)^{2}\right] \\
& +\operatorname{PrEc} F M^{2}\left(u_{i}^{2}+w_{i}^{2}\right)=0,
\end{aligned}
$$

where $A=b m n h^{2}, B=m s h^{2}, C=m h^{2}, D=(K / m), F=$ $K h^{2} s$, and $A, B, C, D$, and $F$ are all equal to 1 for phase I.

The nondimensional forms of the boundary and interface conditions (2) become

$$
\begin{aligned}
& u_{1}(1)=0, \\
& w_{1}(1)=0 ; \\
& u_{1}(0)=u_{2}(0),
\end{aligned}
$$

$$
\begin{aligned}
w_{1}(0) & =w_{2}(0) \\
u_{2}(-1) & =0 \\
w_{2}(-1) & =0 \\
\left(\frac{d u_{1}}{d y}\right) & =\left(\frac{1}{m h}\right)\left(\frac{d u_{2}}{d y}\right), \\
\left(\frac{d w_{1}}{d y}\right) & =\left(\frac{1}{m h}\right)\left(\frac{d w_{2}}{d y}\right) \quad \text { at } y=0 \\
\theta_{1}(1) & =1, \\
\theta_{1}(0) & =\theta_{2}(0), \\
\theta_{2}(-1) & =0, \\
\left(\frac{d \theta_{1}}{d y}\right) & =\left(\frac{1}{K h}\right)\left(\frac{d \theta_{2}}{d y}\right) \quad \text { at } y=0 .
\end{aligned}
$$

The asterisks have been dropped for simplicity. Further writing $q_{1}=u_{1}+i w_{1}$ and $q_{2}=u_{2}+i w_{2}$, (4) can be written in complex form as

$$
\begin{aligned}
& \left(\frac{d^{2} q_{i}}{d y^{2}}\right)+\left(\frac{\mathrm{Gr}}{\operatorname{Re}}\right) A \sin \phi \theta_{i}-B M^{2} q_{i}=\mathrm{CP}-2 i R^{2} q_{i} \\
& \left(\frac{d^{2} \theta_{i}}{d y^{2}}\right)+\operatorname{PrEc} D\left[\left(\frac{d q_{i}}{d y}\right)\left(\frac{d \bar{q}_{i}}{d y}\right)\right] \\
& \quad+\operatorname{PrEc} F M^{2}\left(q_{i} \bar{q}_{i}\right)=0
\end{aligned}
$$

which are to be solved subject to the boundary and interface conditions:

$$
\begin{aligned}
q_{1}(1) & =0, \\
q_{1}(0) & =q_{2}(0), \\
q_{2}(-1) & =0, \\
\left(\frac{d q_{1}}{d y}\right) & =\left(\frac{1}{m h}\right)\left(\frac{d q_{2}}{d y}\right) \quad \text { at } y=0, \\
\theta_{1}(1) & =1, \\
\theta_{1}(0) & =\theta_{2}(0), \\
\theta_{2}(-1) & =0, \\
\left(\frac{d \theta_{1}}{d y}\right) & =\left(\frac{1}{K h}\right)\left(\frac{d \theta_{2}}{d y}\right) \quad \text { at } y=0 .
\end{aligned}
$$

\section{Solutions}

The governing equations of momentum (6) along with the energy equations (7) are to be solved subject to the boundary and interface conditions (8) for the velocity and temperature distributions. Here, we consider the Eckert number very small. Hence, the product $\operatorname{PrEc}(=\varepsilon)$ is very small and can 
be exploited to use the regular perturbation method. The solutions are assumed in the form

$$
\left(q_{i}, \theta_{i}\right)=\left(q_{i 0}, \theta_{i 0}\right)+\varepsilon\left(q_{i 1}, \theta_{i 1}\right)+\cdots,
$$

where $q_{i 0}, \theta_{i 0}$ are solutions for the case $\varepsilon$ equal to zero. $q_{i 1}, \theta_{i 1}$ are perturbed quantities relating to $q_{i 0}, \theta_{i 0}$, respectively. Substituting the above solution in (6) and (7) and equating the coefficients of similar powers of $\varepsilon$ to zero, we get the zeroth and the first order equations as follows.

Zeroth order equations:

$$
\begin{gathered}
\left(\frac{d^{2} q_{i 0}}{d y^{2}}\right)+\left(\frac{\mathrm{Gr}}{\mathrm{Re}}\right) A \sin \phi \theta_{i 0}-B M^{2} q_{i 0} \\
=\mathrm{CP}-2 i R^{2} q_{i 0}, \\
\left(\frac{d^{2} \theta_{i 0}}{d y^{2}}\right)=0 .
\end{gathered}
$$

First order equations:

$$
\begin{aligned}
& \left(\frac{d^{2} q_{i 1}}{d y^{2}}\right)+\left(\frac{\mathrm{Gr}}{\mathrm{Re}}\right) A \sin \phi \theta_{i 1}-B M^{2} q_{i 1} \\
& =-2 i R^{2} q_{i 1} \\
& \left(\frac{d^{2} \theta_{i 1}}{d y^{2}}\right)+D\left[\left(\frac{d q_{i 0}}{d y}\right)\left(\frac{d \bar{q}_{i 0}}{d y}\right)\right]+F M^{2}\left(q_{i 0} \bar{q}_{i 0}\right) \\
& =0
\end{aligned}
$$

The corresponding Boundary conditions (8) reduce to

$$
\begin{aligned}
q_{10}(1) & =0 \\
q_{10}(0) & =q_{20}(0), \\
q_{20}(-1) & =0, \\
\left(\frac{d q_{10}}{d y}\right) & =\left(\frac{1}{m h}\right)\left(\frac{d q_{20}}{d y}\right) \quad \text { at } y=0, \\
\theta_{10}(1) & =1 \\
\theta_{10}(0) & =\theta_{20}(0), \\
\theta_{20}(-1) & =0 \\
\left(\frac{d \theta_{10}}{d y}\right) & =\left(\frac{1}{K h}\right)\left(\frac{d \theta_{20}}{d y}\right) \quad \text { at } y=0, \\
q_{11}(1) & =0 \\
q_{11}(0) & =q_{21}(0), \\
q_{21}(-1) & =0
\end{aligned}
$$

$$
\begin{aligned}
\left(\frac{d q_{11}}{d y}\right) & =\left(\frac{1}{m h}\right)\left(\frac{d q_{21}}{d y}\right) \quad \text { at } y=0, \\
\theta_{11}(1) & =0, \\
\theta_{11}(0) & =\theta_{21}(0), \\
\theta_{21}(-1) & =0, \\
\left(\frac{d \theta_{11}}{d y}\right) & =\left(\frac{1}{K h}\right)\left(\frac{d \theta_{21}}{d y}\right) \quad \text { at } y=0 .
\end{aligned}
$$

We observe that (10) and (11) are linear and coupled and therefore can be solved exactly. Here, we consider $q_{10}=u_{10}+$ $i w_{10}, q_{20}=u_{20}+i w_{20}, q_{11}=u_{11}+i w_{11}$, and $q_{21}=u_{21}+i w_{21}$.

Solutions of the zeroth order equations (10) using boundary conditions (12) are

$$
\begin{gathered}
\theta_{10}=\frac{(y+K h)}{(1+K h)}, \\
\theta_{20}=\frac{(1+y) K h}{(1+K h)}, \\
u_{10}=\left[\left(c_{1} e^{A_{5} y}+c_{2} e^{-A_{5} \cdot y}\right) \cos \left(A_{6} y\right)+A_{14}+A_{12} y\right], \\
w_{10} \\
=-\left[\left(c_{1} e^{A_{5} y}-c_{2} e^{-A_{5} \cdot y}\right) \sin \left(A_{6} y\right)-A_{15}-A_{13} y\right], \\
u_{20}=\left[\left(c_{3} e^{A_{23} y}+c_{4} e^{-A_{23} \cdot y}\right) \cos \left(A_{24} y\right)+A_{30}+A_{32} y\right], \\
w_{20}=-\left[\left(c_{3} e^{A_{23} y}-c_{4} e^{-A_{23} \cdot y}\right) \sin \left(A_{24} y\right)-A_{31}-A_{33} y\right] .
\end{gathered}
$$

Solutions of the first order equations (11) using boundary conditions (13) are

$$
\begin{aligned}
\theta_{11}=\{ & c_{5} y+c_{6}+B_{56} e^{2 A_{5} y}+B_{57} \cos 2 A_{6} y \\
& +B_{58} e^{-2 A_{5} y}+B_{132} e^{A_{5} y} \sin A_{6} y+B_{98} y^{2} \\
& +B_{133} e^{A_{5} y} \cos A_{6} y+B_{134} e^{-A_{5} y} \sin A_{6} y \\
& +B_{135} e^{-A_{5} y} \cos A_{6} y+B_{136} e^{A_{5} y} y \cos A_{6} y \\
& +B_{137} e^{A_{5} y} y \sin A_{6} y+B_{138} e^{-A_{5} y} y \cos A_{6} y \\
& \left.+B_{139} e^{-A_{5} y} y \sin A_{6} y+B_{96} y^{3}+B_{97} y^{4}\right\} \\
+ & i\left\{B_{99} \sin 2 A_{6} y+B_{140} e^{A_{5} y} \sin A_{6} y\right. \\
& +B_{141} e^{A_{5} y} \cos A_{6} y+B_{142} e^{-A_{5} y} \sin A_{6} y
\end{aligned}
$$




$$
\begin{aligned}
& +B_{143} e^{-A_{5} y} \cos A_{6} y+B_{144} e^{A_{5} y} y \cos A_{6} y \\
& +B_{145} e^{A_{5} y} y \sin A_{6} y+B_{146} e^{-A_{5} y} y \cos A_{6} y \\
& \left.+B_{147} e^{-A_{5} y} y \sin A_{6} y\right\} \text {, } \\
& \theta_{21}=\left\{c_{7} y+c_{8}+E_{67} e^{2 A_{23} y}+E_{68} \cos 2 A_{24} y\right. \\
& +E_{69} e^{-2 A_{23} y}+E_{135} e^{A_{23} y} \sin A_{24} y \\
& +E_{136} e^{A_{23} y} \cos A_{24} y+E_{137} e^{-A_{23} y} \sin A_{24} y \\
& +E_{138} e^{-A_{23} y} \cos A_{24} y-E_{107} y^{4}-E_{108} y^{3} \\
& +E_{139} e^{A_{23} y} y \cos A_{24} y+E_{140} e^{A_{23} y} y \sin A_{24} y \\
& +E_{141} e^{-A_{23} y} y \cos A_{24} y-E_{109} y^{2} \\
& \left.+E_{142} e^{-A_{23} y} y \sin A_{24} y\right\} \\
& +i\left\{E_{110} \sin 2 A_{24} y+E_{143} e^{A_{23} y} \sin A_{24} y\right. \\
& +E_{144} e^{A_{23} y} \cos A_{24} y+E_{145} e^{-A_{23} y} \sin A_{24} y \\
& +E_{146} e^{-A_{23} y} \cos A_{24} y+E_{147} e^{A_{23} y} y \cos A_{24} y \\
& +E_{148} e^{A_{23} y} y \sin A_{24} y+E_{149} e^{-A_{23} y} y \cos A_{24} y \\
& \left.+E_{150} e^{-A_{23} y} y \sin A_{24} y\right\} \text {, } \\
& u_{11}=\left(c_{9} e^{A_{5} y}+c_{10} e^{-A_{5} y}\right) \cos A_{6} y \\
& +F_{95} e^{2 A_{5} y}+F_{96} e^{-2 A_{5} y}-F_{97} \cos 2 A_{6} y \\
& +F_{98} \sin 2 A_{6} y+F_{99} e^{A_{5} y} y \sin A_{6} y \\
& +F_{100} e^{A_{5} y} y \cos A_{6} y-F_{101} e^{-A_{5} y} y \cos A_{6} y \\
& -F_{107} y^{4}-F_{108} y^{3}+F_{102} e^{-A_{5} y} y \sin A_{6} y \\
& +F_{103} e^{A_{5} y} y^{2} \sin A_{6} y+F_{104} e^{A_{5} y} y^{2} \cos A_{6} y \\
& -F_{109} y^{2}-F_{110} y+F_{105} e^{-A_{5} y} y^{2} \sin A_{6} y \\
& -F_{106} e^{-A_{5} y} y^{2} \cos A_{6} y-F_{111} \\
& w_{11}=\left(c_{10} e^{-A_{5} y}-c_{9} e^{A_{5} y}\right) \sin A_{6} y \\
& -F_{112} e^{2 A_{5} y}-F_{113} e^{-2 A_{5} y}-F_{114} \cos 2 A_{6} y \\
& -F_{115} \sin 2 A_{6} y+F_{116} e^{A_{5} y} y \cos A_{6} y \\
& +F_{117} e^{A_{5} y} y \sin A_{6} y-F_{125} y^{3}-F_{126} y^{2} \\
& -F_{118} e^{-A_{5} y} y \cos A_{6} y+F_{119} e^{-A_{5} y} y \sin A_{6} y \\
& +F_{120} e^{A_{5} y} y^{2} \sin A_{6} y-F_{127} y-F_{128} \\
& +F_{121} e^{A_{5} y} y^{2} \cos A_{6} y+F_{122} e^{-A_{5} y} y^{2} \sin A_{6} y \\
& -F_{123} e^{-A_{5} y} y^{2} \cos A_{6} y-F_{124} y^{4} \text {, }
\end{aligned}
$$

$$
\begin{aligned}
& u_{21}=\left(c_{11} e^{A_{23} y}+c_{12} e^{-A_{23} y}\right) \cos A_{24} y \\
&+G_{96} e^{2 A_{23} y}+G_{97} e^{-2 A_{23} y}+G_{98} \cos 2 A_{24} y \\
&+G_{112}-G_{99} \sin 2 A_{24} y+G_{100} e^{A_{23} y} y \sin A_{24} y \\
&+G_{101} e^{A_{23} y} y \cos A_{24} y+G_{108} y^{4}+G_{109} y^{3} \\
&+G_{102} e^{-A_{23} y} y \cos A_{24} y+G_{103} e^{-A_{23} y} y \sin A_{24} y \\
&+G_{104} e^{A_{23} y} y^{2} \sin A_{24} y+G_{110} y^{2} \\
&+G_{105} e^{A_{23} y} y^{2} \cos A_{24} y+G_{106} e^{-A_{23} y} y^{2} \cos A_{24} y \\
&+G_{107} e^{-A_{23} y} y^{2} \sin A_{24} y+G_{111} y \\
&=\left(c_{12} e^{-A_{23} y}-c_{11} e^{A_{23} y}\right) \sin A_{24} y \\
&-G_{113} e^{2 A_{23} y}-G_{114} e^{-2 A_{23} y}+G_{115} \cos 2 A_{24} y \\
&+G_{129}+G_{116} \sin 2 A_{24} y+G_{117} e^{A_{23} y} y \sin A_{24} y \\
&+G_{124} e^{-A_{23} y} y^{2} \cos A_{24}+G_{125} y^{4} \cdot \\
&+G_{122} e^{A_{23} y} y \cos A_{24} y+G_{128} y+G_{127} y^{2} \\
&+G_{119} e^{-A_{23} y} y \cos A_{24} y+G_{120} e^{-A_{23} y} y \sin A_{24} y \\
&+G_{121} e^{A_{23} y} y^{2} \sin A_{24} y+G_{126} y^{3} \\
& \\
& \\
& w_{21} A_{24} y+G_{123} e^{-A_{23} y} y^{2} \sin A_{24} y \\
&
\end{aligned}
$$

The constants appearing in (15) and (16) are not given for the sake of brevity. Since the problem contains too many nondimensional parameters, for the sake of conciseness, we fix $P=-5.0, b=1.0, \operatorname{Re}=5.0, n=1.5$, and $K=1.0$. In the figures, all the other parameters except the varying one are chosen from the set $(M, \mathrm{Gr}, \phi, m, h, s, R)=\left(2.0,5.0,30^{\circ}, 0.5\right.$, $1.0,2.0,2.0)$.

\section{Results and Discussion}

Two-phase magnetohydrodynamic convective flow between two infinite inclined parallel plates in a rotating system is studied analytically. The resulting differential equations are solved using perturbation method to obtain approximate solutions for temperature distribution and primary and secondary velocity distributions. Here, we note that when $R=0$, that is, in the absence of rotation, these results are in agreement with that of Malashetty et al. [16].

The effect of rotation parameter $R$ on primary velocity $u$ and secondary velocity $w$ is shown in Figures 2 and 3, respectively. From Figure 2, it is observed that the primary velocity $u$ decreases with the increase in the rotation parameter. The rotation parameter $R$ defines the relative magnitude of the Coriolis force and the viscous force in the regime. As the high magnitude Coriolis forces oppose the buoyancy force, the velocity will be decreased. From Figure 3, it is concluded that as the rotation parameter $R$ increases in $(0,2)$, the secondary 


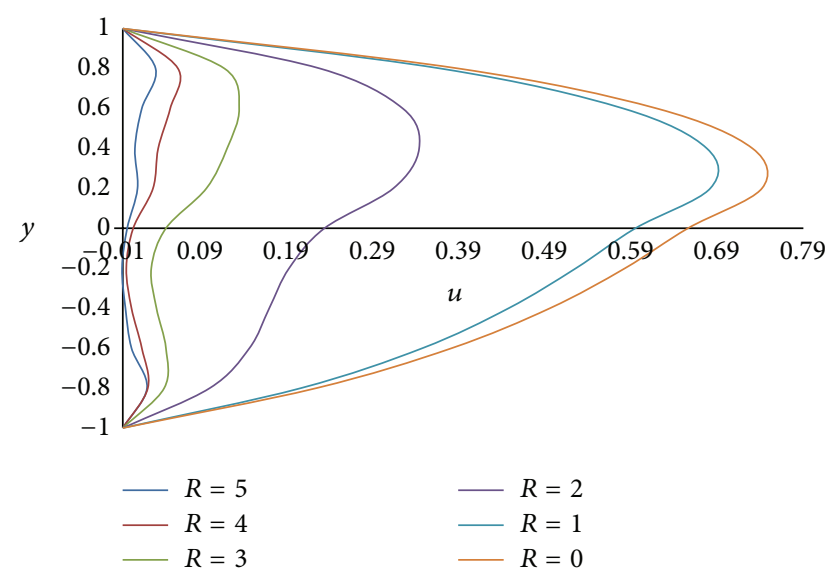

Figure 2: Primary velocity profiles for different values of rotation parameter $R$.

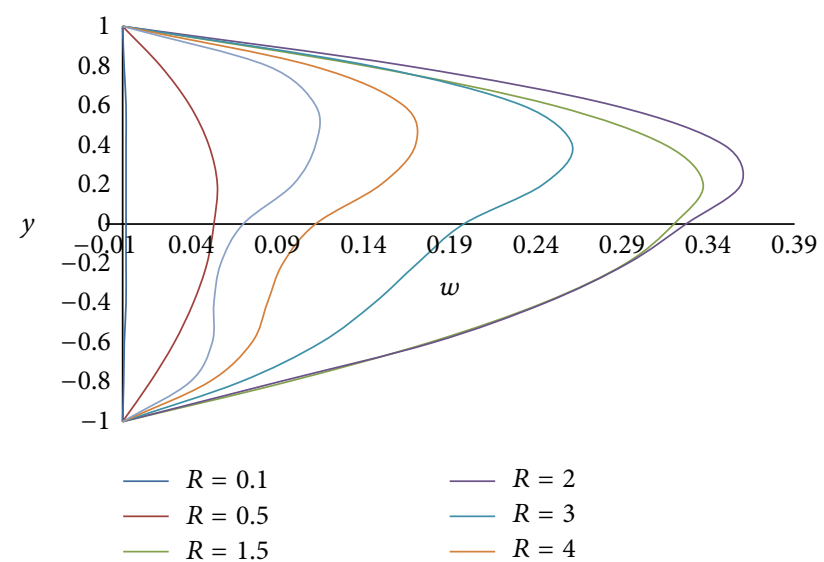

FIGURE 3: Secondary velocity profiles for different values of rotation parameter $R$.

velocity $w$ also increases but outside this range as $R$ increases, it decreases. Therefore, by increasing the rotation parameter $R$, the secondary flow becomes oscillatory.

Figures 4 and 5 show the effect of the angle of inclination $\phi$ on primary velocity $u$ and secondary velocity $w$, respectively. As the angle of inclination $\phi$ increases, both the primary and secondary velocities increase because the magnitude of the buoyancy force increases with increase in the inclination angle. The effect of the ratio of the viscosities $m$ on primary and secondary velocities is shown in Figures 6 and 7 , respectively. The smaller the value of the viscosity of the fluid in the lower phase compared to the fluid in the upper phase, the larger the primary and secondary flow fields.

Figures 8 and 9 represent the effect of the ratio of heights $h$ on primary and secondary velocities, respectively. The smaller the height of the upper phase compared to the lower phase, the larger the primary as well as secondary flow fields. The effect of the ratio of the electrical conductivity $s$ on primary velocity $u$ and secondary velocity $w$ is shown in Figures 10 and 11, respectively. We have observed that as the

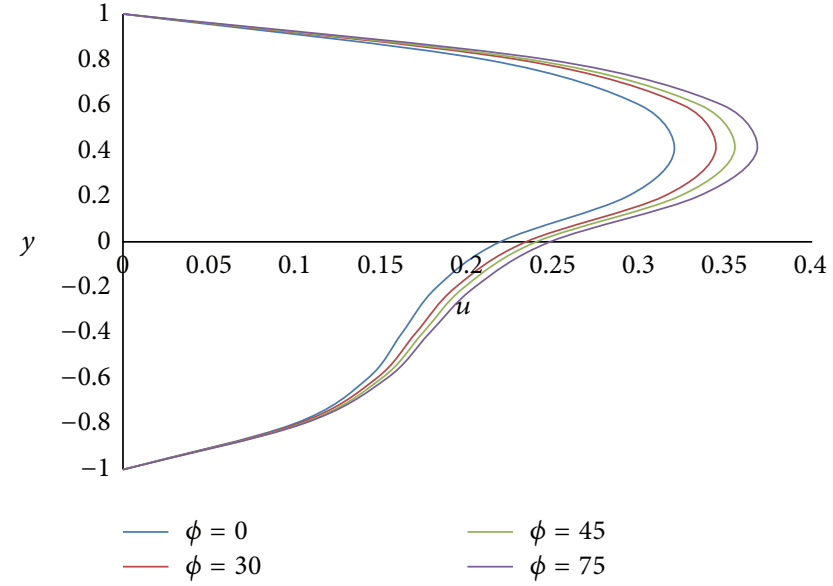

FIGURE 4: Primary velocity profiles for different values of inclination angle $\phi$.

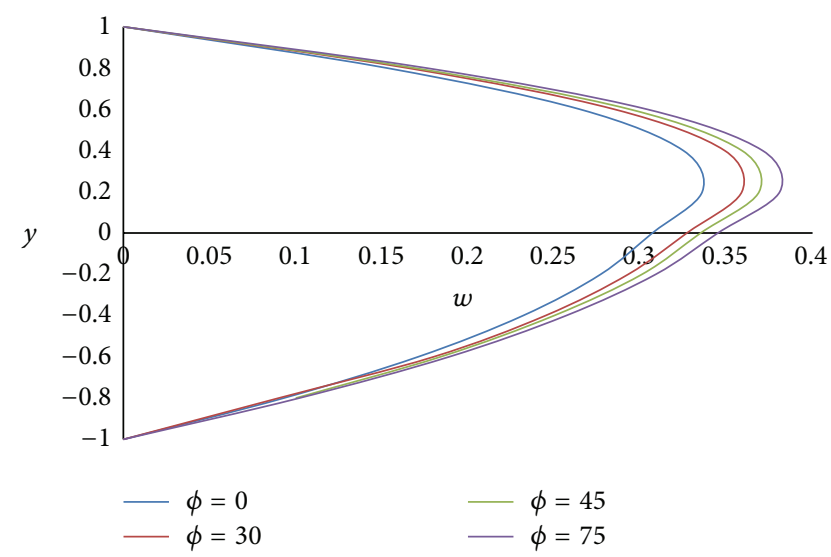

FIGURE 5: Secondary velocity profiles for different values of inclination angle $\phi$.

ratio of the electrical conductivity $s$ increases, the primary velocity $u$ increases but the secondary velocity $w$ decreases.

Figures 12 and 13 show the effect of Hartmann number $M$ on primary and secondary velocities, respectively. The effect of increasing Hartmann number $M$ is to decrease both the primary and secondary velocities. This is because an increase in applied magnetic field strength causes greater interaction between the fluid motion and the magnetic field, therefore, an increase in the Lorentz force. Since this force opposes the buoyancy force, both the velocities will be decreased. The effect of Grashof number Gr on primary and secondary velocities is shown in Figures 14 and 15, respectively. We find that an increase in the value of Grashof number Gr increases both the primary and secondary velocities.

The effect of rotation parameter on temperature $\theta$ can be seen in Figure 16. From the figure, it is evident that the temperature decreases with the increase in the rotation parameter $R$. The rotation parameter $R\left(R^{2}=\Omega h_{i}^{2} / \nu\right)$ defines the relative magnitude of the Coriolis force and the viscous force in the regime. As the high magnitude Coriolis forces oppose the buoyancy force, the velocity will be decreased 


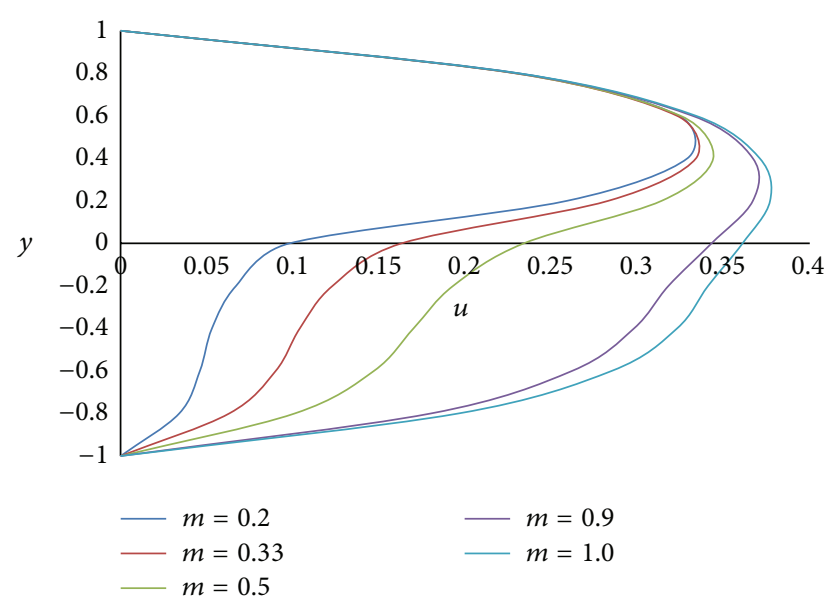

Figure 6: Primary velocity profiles for different values of ratio of viscosities $m$.

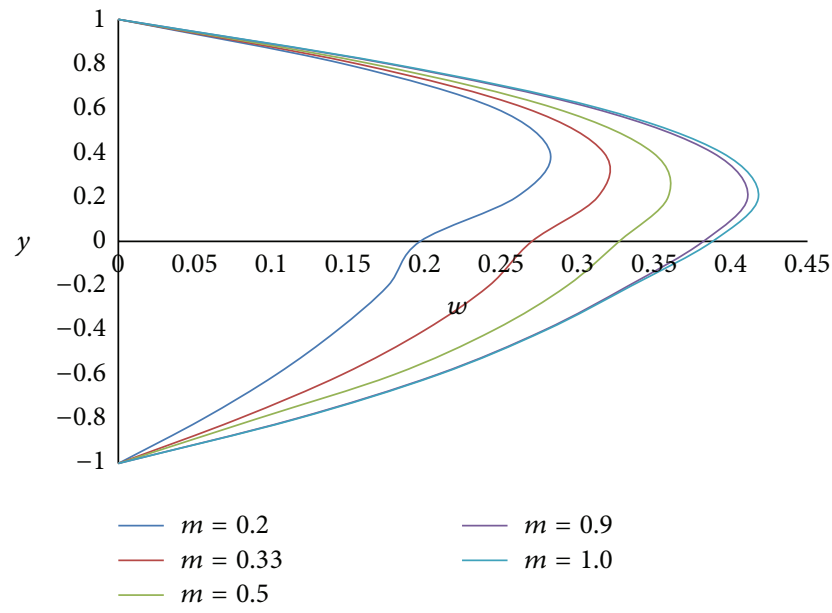

FIGURE 7: Secondary velocity profiles for different values of ratio of viscosities $m$.

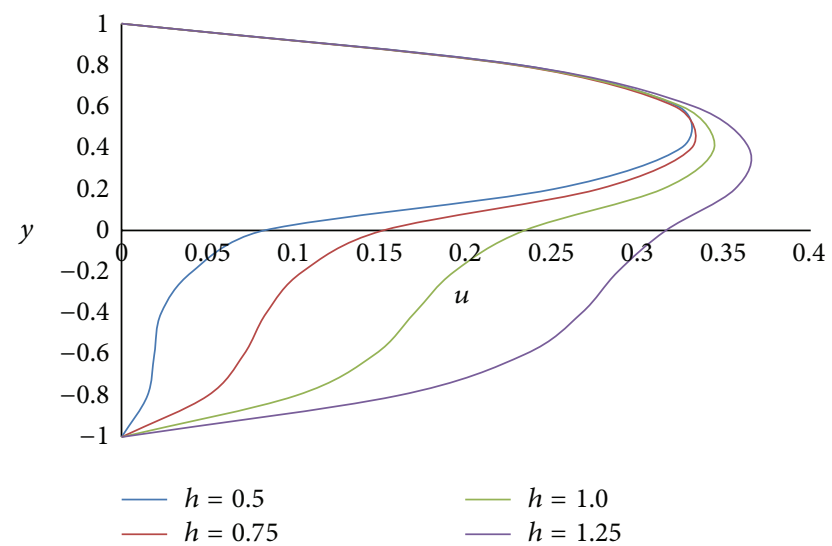

FIGURE 8: Primary velocity profiles for different values of ratio of heights $h$.

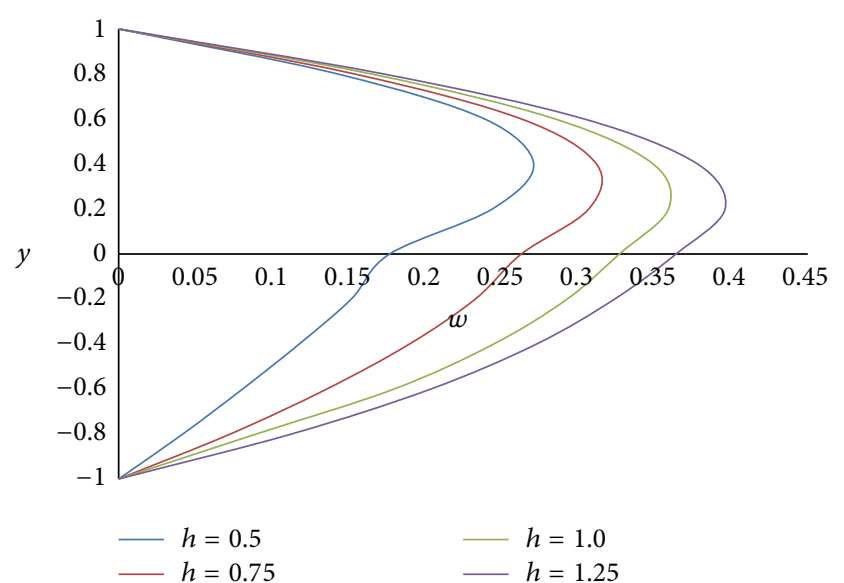

FIGURE 9: Secondary velocity profiles for different values of ratio of heights $h$.

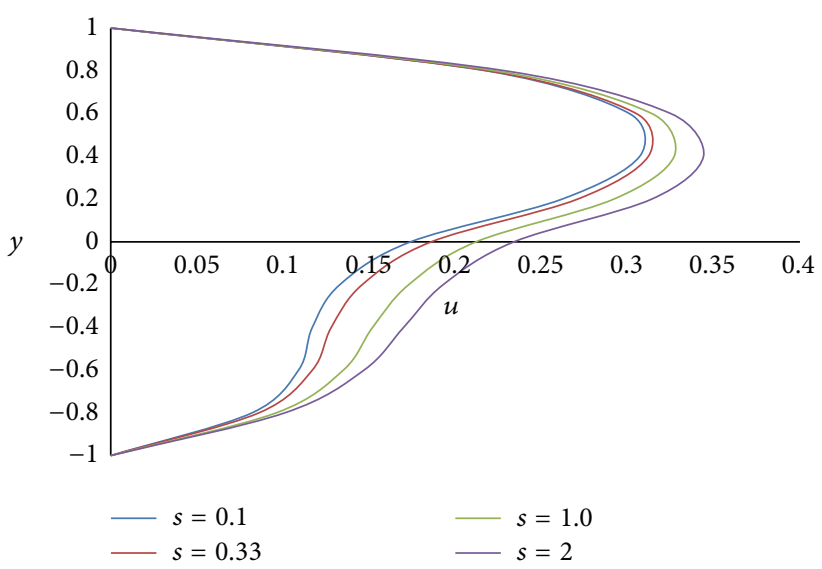

FIgURE 10: Primary velocity profiles for different values of ratio of electrical conductivities $s$.

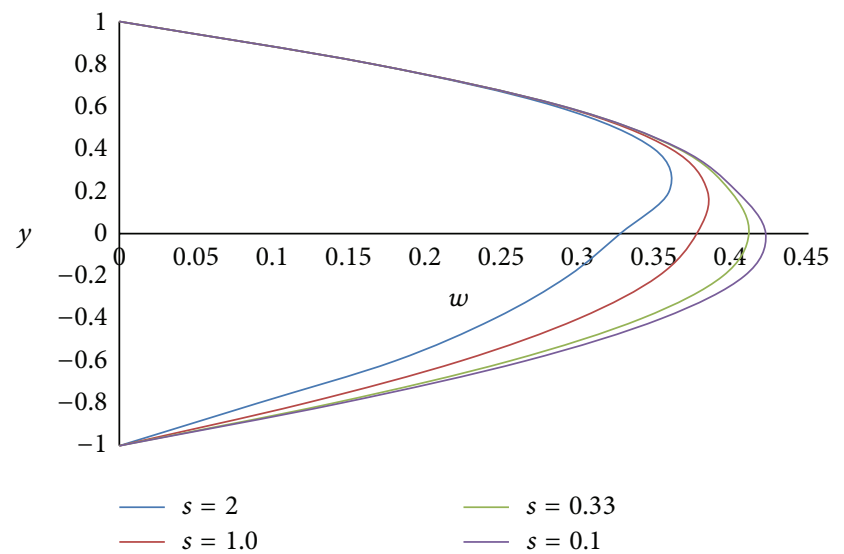

FIGURE 11: Secondary velocity profiles for different values of ratio of electrical conductivities $s$. 


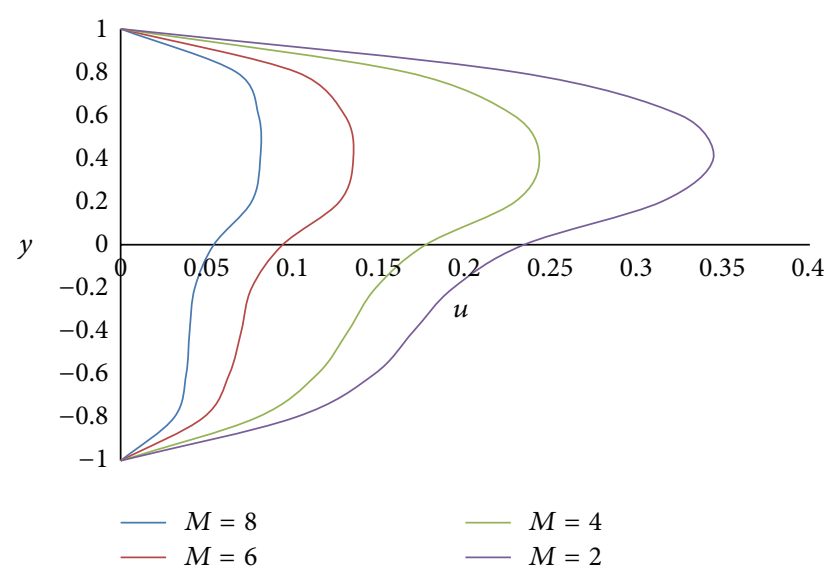

FIGURE 12: Primary velocity profiles for different values of Hartmann number $M$.

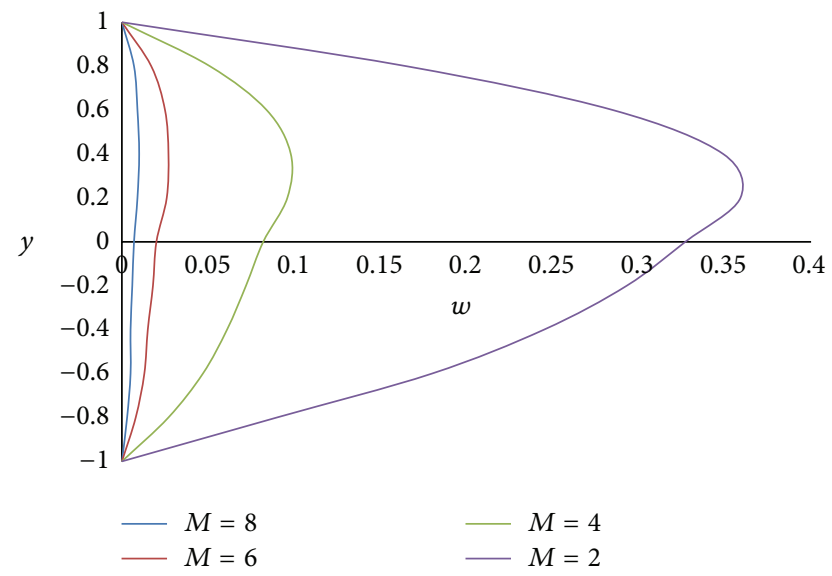

FIGURE 13: Secondary velocity profiles for different values of Hartmann number $M$.

leading to a reduction in the viscous and Joule dissipation and so to a reduction in the temperature.

Figure 17 shows the effect of the angle of inclination $\phi$ on temperature $\theta$. As the angle of inclination $\phi$ increases, the temperature $\theta$ also increases because the magnitude of the buoyancy force increases with the increase in the inclination angle. The effect of the ratio of viscosities $m$ on the temperature $\theta$ is shown in Figure 18. From the figure, it is observed that the less viscous fluid in the lower phase adds the heat transfer. Figure 19 exhibits the effect of the ratio of heights $h$ on the temperature $\theta$. From the figure, it is noticed that the smaller the height of the upper phase compared to the lower phase, the larger the magnitude of the temperature. Figure 20 shows the effect of the ratio of electrical conductivities $s$ on the temperature $\theta$. From the figure, it is concluded that as the ratio of electrical conductivities $s$ increases, the temperature also increases.

Figure 21 represents the effect of Hartmann number $M$ on the temperature $\theta$. From the figure, it is clear that the effect of increasing $M$ is to decrease the temperature. This is because an increase in the applied magnetic field strength

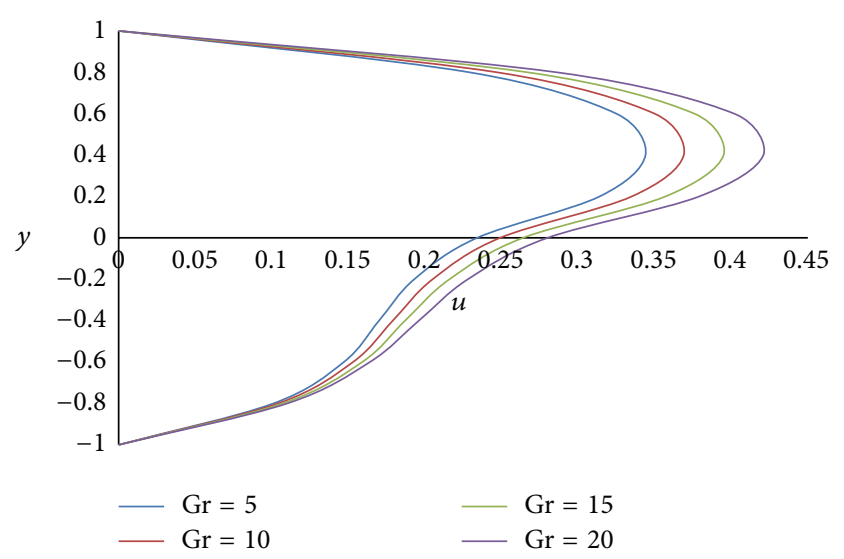

Figure 14: Primary velocity profiles for different values of Grashof number Gr.

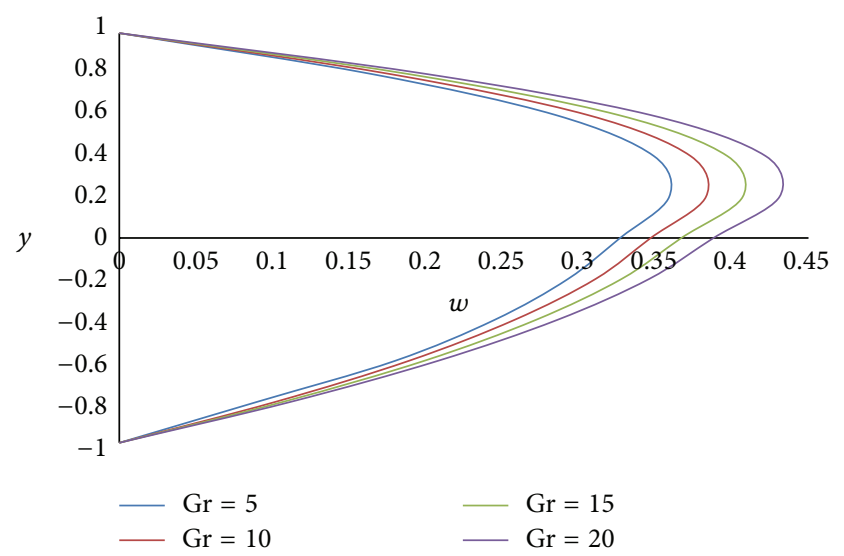

FIGURE 15: Secondary velocity profiles for different values of Grashof number Gr.

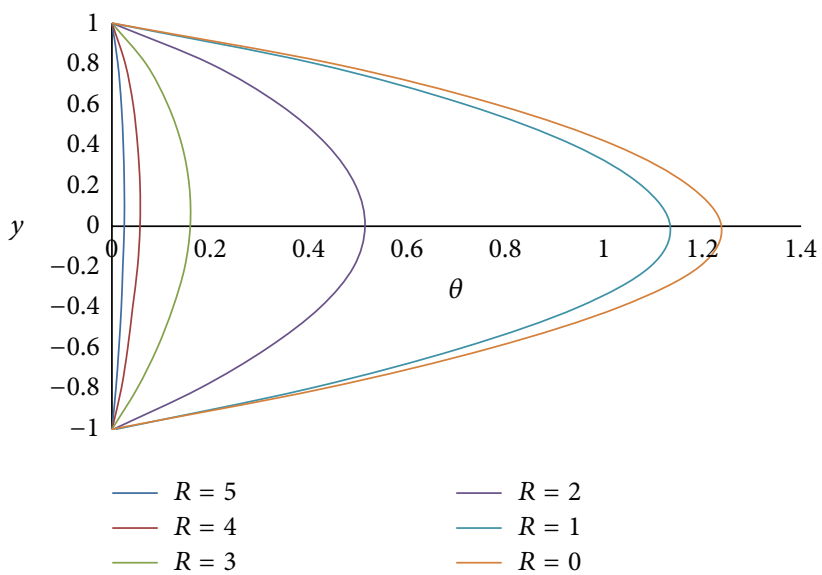

FIGURE 16: Temperature profiles for different values of rotation parameter $R$. 


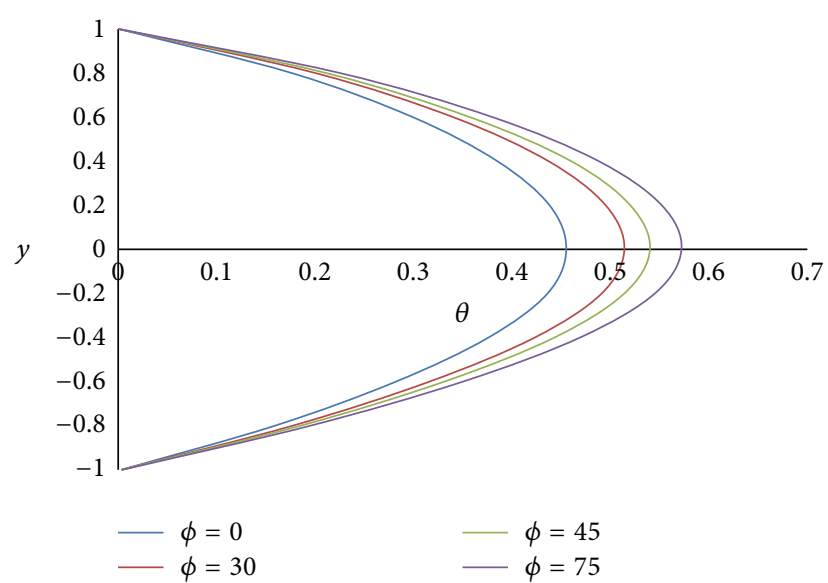

FIGURE 17: Temperature profiles for different values of inclination angle $\phi$.

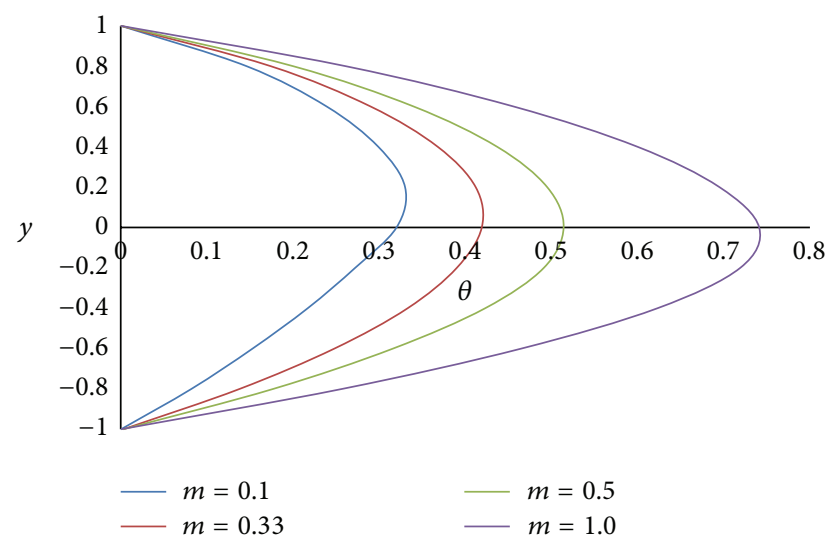

FigURE 18: Temperature profiles for different values of ratio of viscosities $m$.

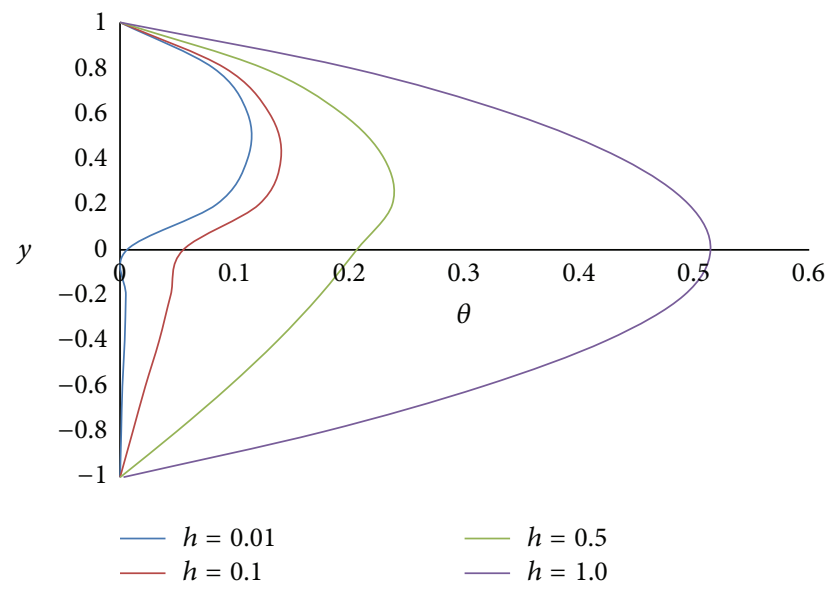

Figure 19: Temperature profiles for different values of ratio of heights $h$.

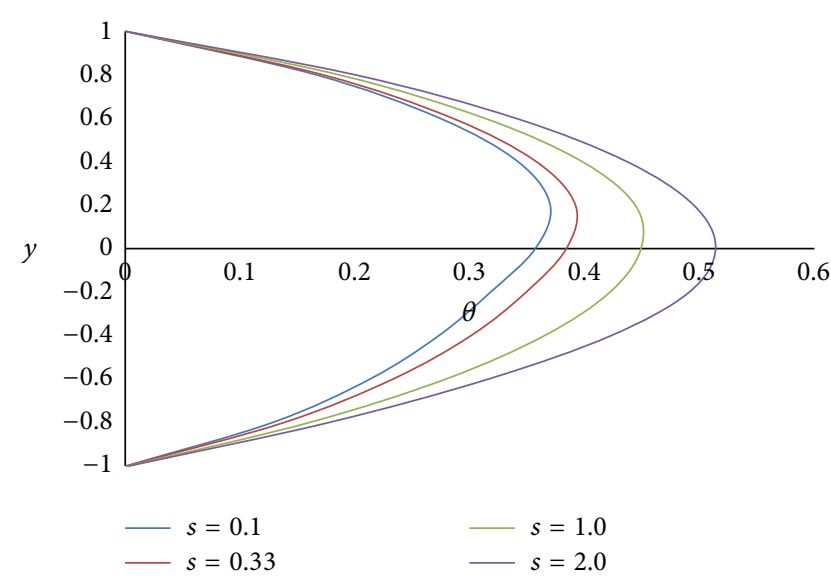

FIgURE 20: Temperature profiles for different values of ratio of electrical conductivities $s$.

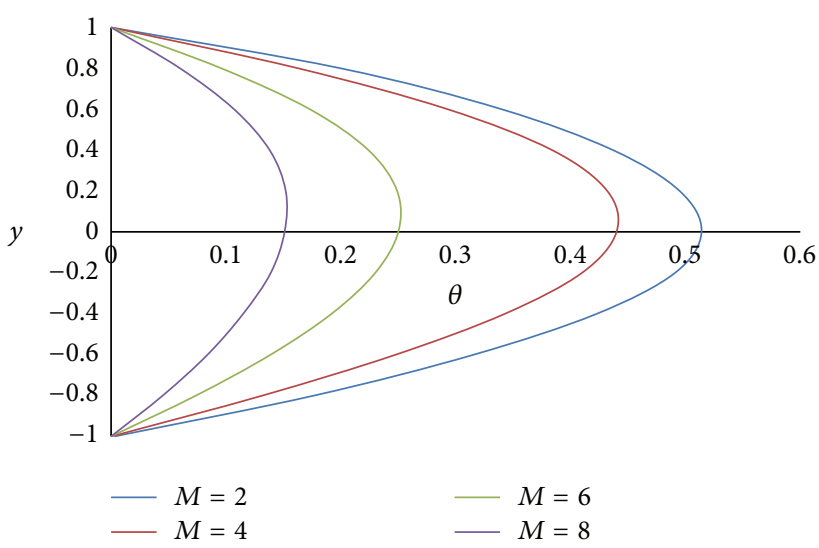

FIGURE 21: Temperature profiles for different values of Hartmann number $M$.

causes greater interaction between the fluid motion and the magnetic field, therefore, an increase in the Lorentz force. Since this force opposes the buoyancy force, the temperature will be decreased. The effect of Grashof number Gr on temperature $\theta$ is shown in Figure 22; we observe from this figure that an increase in the value of Grashof number $\mathrm{Gr}$ increases the temperature $\theta$.

\section{Nomenclature}

$B_{0}$ : Magnetic field strength

$b$ : Ratio of the coefficients of thermal expansion $\left(\beta_{2} / \beta_{1}\right)$

$C_{p}$ : Specific heat at constant pressure

Ec: Eckert number $\left[\left(\bar{u}_{1}\right)^{2} /\left(C_{p} \Delta T\right)\right]$

$g$ : Acceleration due to gravity

$h$ : Ratio of the heights of the two phases $\left(h_{2} / h_{1}\right)$

Gr: Grashof number $\left[g \beta_{1} h_{1}^{3} \Delta T / \nu_{1}^{2}\right]$

$K$ : Ratio of the thermal conductivities $\left(K_{1} / K_{2}\right)$ 


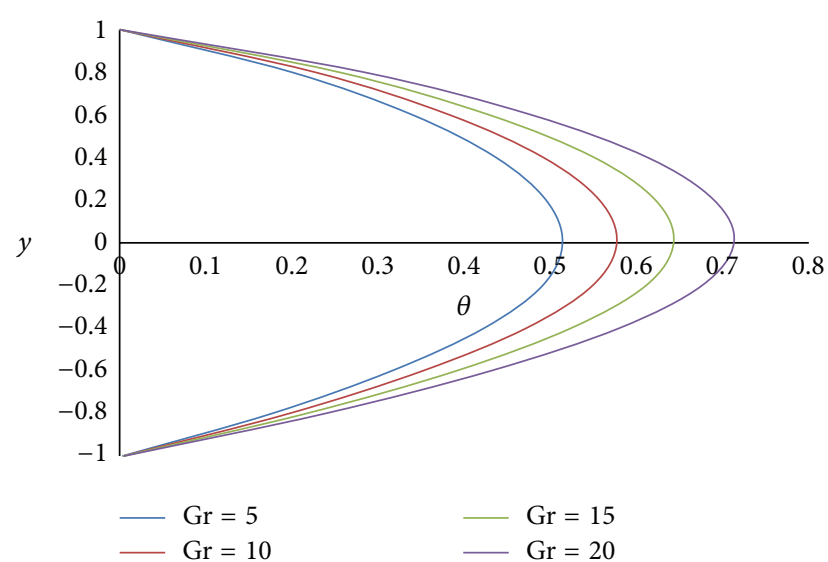

FIGURE 22: Temperature profiles for different values of Grashof number Gr.

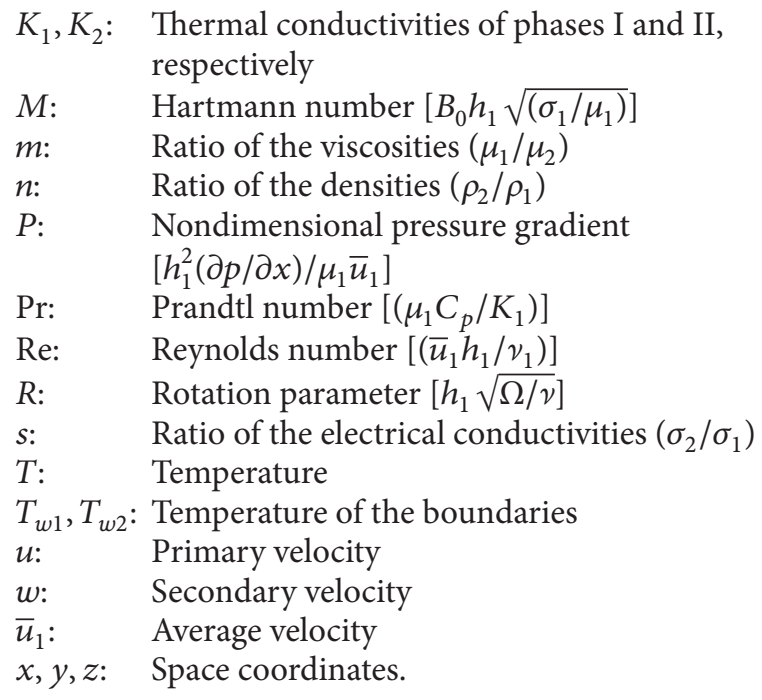

\section{Greek Symbols}

$\beta$ : Coefficient of thermal expansion

$\sigma$ : $\quad$ Electrical conductivity

$\phi$ : Angle of inclination

$\rho:$ Density

$\nu$ : Kinematic viscosity

$\mu$ : Viscosity

$\varepsilon$ : Product of Prandtl number and Eckert number $(\mathrm{Pr} \cdot \mathrm{Ec})$

$\Delta T$ : Difference in temperature $\left[T_{w 1}-T_{w 2}\right]$

$\theta: \quad$ Nondimensional temperature $\left[\left(T-T_{w 2}\right) / \Delta T\right]$

$\Omega$ : Angular velocity.

Subscript

$i$ : Value for phase.

\section{Conflict of Interests}

The authors have declared that no conflict of interests exists.

\section{References}

[1] R. Shail, "On laminar two-phase flows in magnetohydrodynamics," International Journal of Engineering Science, vol. 11, no. 10, pp. 1103-1108, 1973.

[2] N. Rudraiah, V. Kumudini, and W. Unno, “Theory of nonlinear magnetoconvection and its application to solar convection problems. I, II," Publications of the Astronomical Society of Japan, vol. 37, no. 2, pp. 183-233, 1985.

[3] J. C. Umavathi, "A note on magneto convection in a vertical enclosure," International Journal of Non-Linear Mechanics, vol. 31, no. 3, pp. 371-376, 1996.

[4] J. Lohrasbi and V. Sahai, "Hall current and heat transfer effects on MHD flow in a channel partially filled with a porous medium in a rotating system," Applied Scientific Research, vol. 45, pp. 5366, 1988.

[5] M. S. Malashetty and V. Leela, "Magnetohydrodynamic heat transfer in two fluid flow," in Proceedings of the ASME/AIChE 27th National Heat Transfer conference and Exposition, pp. 2831, July 1991.

[6] M. S. Malashetty and V. Leela, "Magnetohydrodynamic heat transfer in two phase flow," International Journal of Engineering Science, vol. 30, no. 3, pp. 371-377, 1992.

[7] T. L. Raju and P. S. R. Murty, "Hydromagnetic two phase flow and heat transfer through two parallel plates in a rotating system," The journal of the Indian Academy of Mathematics, vol. 2, pp. 343-360, 2006.

[8] G. S. Seth, R. Nandkeolyar, and M. S. Ansari, "Hartmann flow in a rotating system in the presence of inclined magnetic field with hall effects," Tamkang Journal of Science and Engineering, vol. 13, no. 3, pp. 243-252, 2010.

[9] D. S. Chauhan and P. Rastogi, "Heat transfer effects on rotating MHD flow in a channel partially filled with a porous medium in a rotating system," Turkish Journal of Engineering and Environmental Sciences, vol. 33, pp. 167-184, 2009.

[10] D. S. Chauhan and P. Rastogi, "Heat transfer effects on rotating MHD couette flow in a channel partially filled by a porous medium with hall current," Journal of Applied Science \& Engineering, vol. 15, no. 3, pp. 281-290, 2012.

[11] Abdul Mateen, "Magnetohydrodynamic flow and heat transfer of two immiscible fluids through a horizontal channel," International Journal of Current Engineering and Technology, vol. 3, pp. 1952-1956, 2013.

[12] Abdul Mateen, "Transient magnetohydrodynamic flow of two immiscible fluids through a horizontal channel," International Journal of Engineering Research, vol. 3, no. 1, pp. 13-17, 2014.

[13] T. L. Raju and M. Nagavalli, "MHD two-layered unsteady fluid flow and heat transfer through a horizontal channel between parallel plates in a rotating system," International Journal of Applied mechanics and Engineering, vol. 19, no. 1, pp. 97-121, 2014.

[14] P. S. R. Murty and T. Linga Raju, "MHD two-phase flow and heat transfer between two parallel porous walls in a rotating system," Science Domain International, vol. 4, no. 13, pp. 1894-1907, 2014.

[15] M. S. Malashetty and J. C. Umavathi, "Two-phase magnetohydrodynamic flow and heat transfer in an inclined channel," International Journal of Multiphase Flow, vol. 23, no. 3, pp. 545560, 1997.

[16] M. S. Malashetty, J. C. Umavathi, and J. Prathap Kumar, "Convective magnetohydrodynamic two fluid flow and heat transfer in an inclined channel," Heat and Mass Transfer, vol. 37, no. 2-3, pp. 259-264, 2001. 
[17] S. Daniel and Y. S. Daniel, "Convective flow two immiscible fluids and heat transfer with porous along an inclined channel with pressure gradient," Research Inventy: International Journal of Engineering and Science, vol. 2, no. 4, pp. 12-18, 2013.

[18] P. S. R. Murty and G. B. Prakash, "Heat transfer aspects on rotating MHD two-phase convective flow through an inclined channel in the presence of electric field," Physical Science International Journal, Science Domain International, vol. 4, pp. 1260-1279, 2014. 


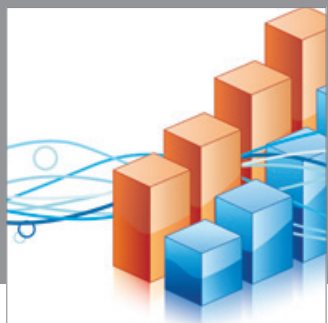

Advances in

Operations Research

mansans

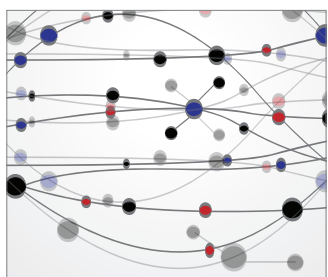

The Scientific World Journal
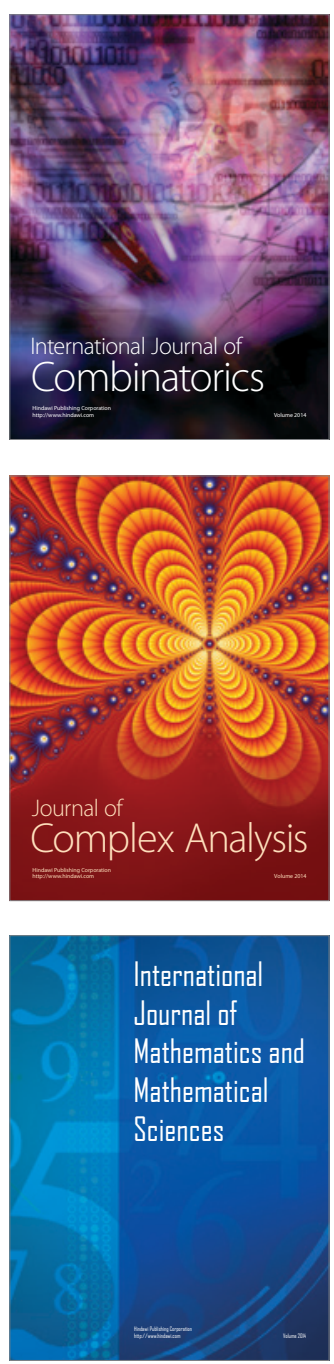
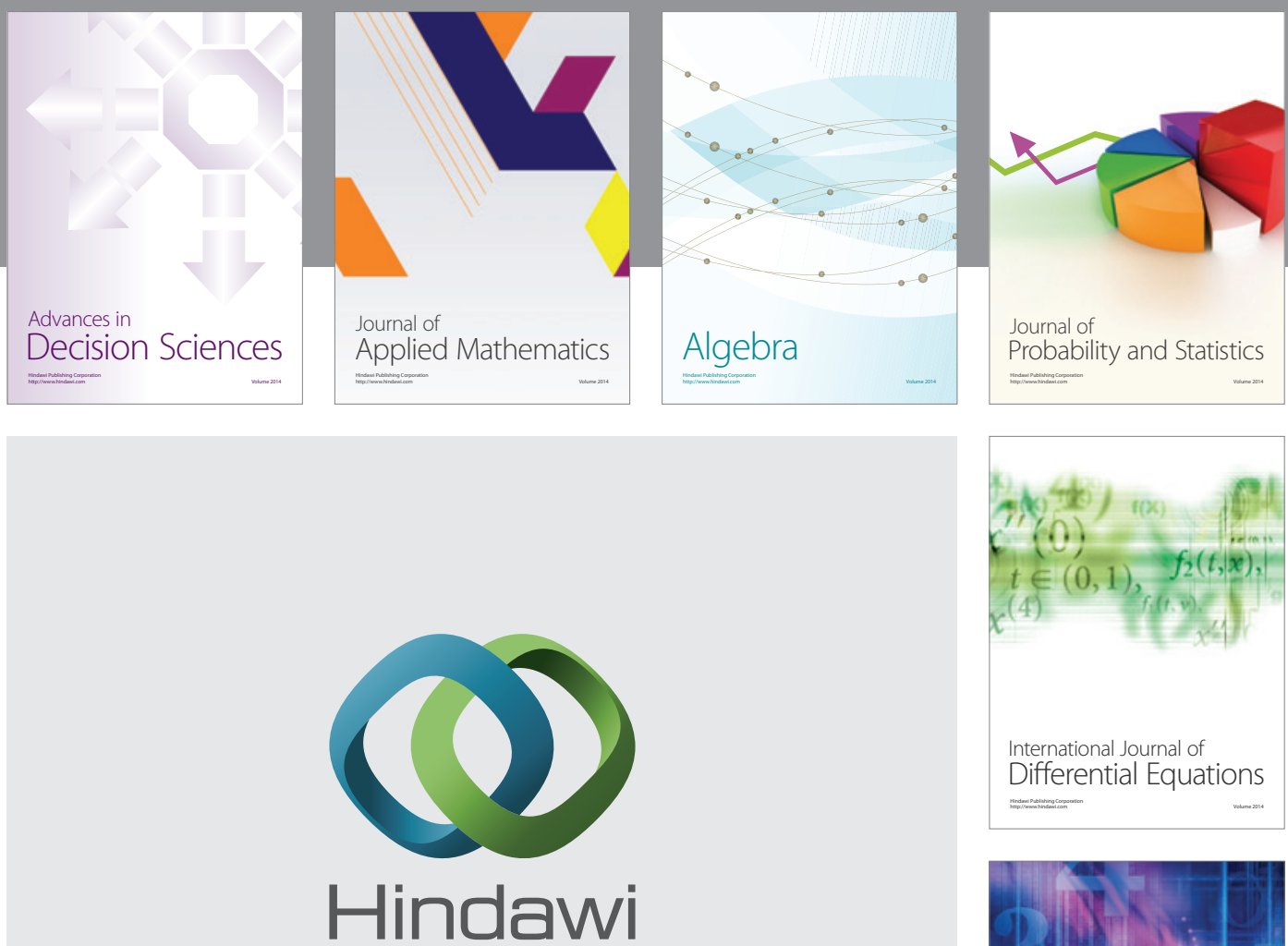

Submit your manuscripts at http://www.hindawi.com
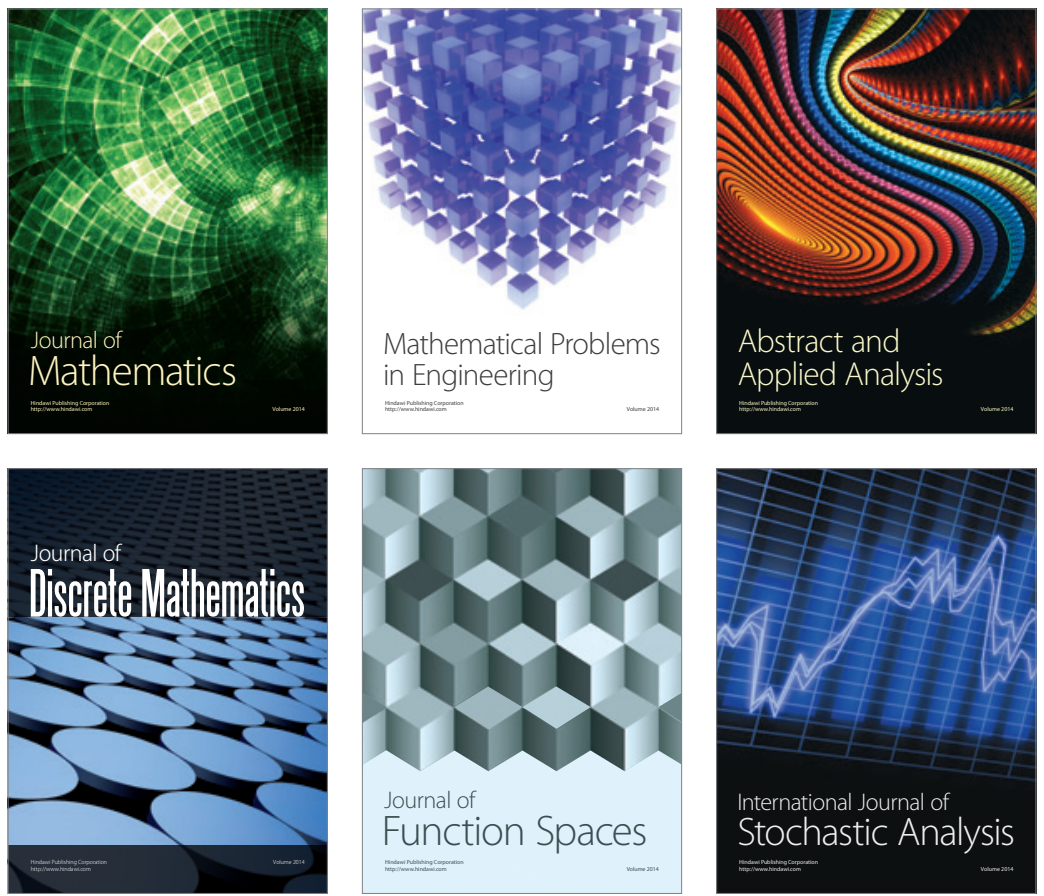

Journal of

Function Spaces

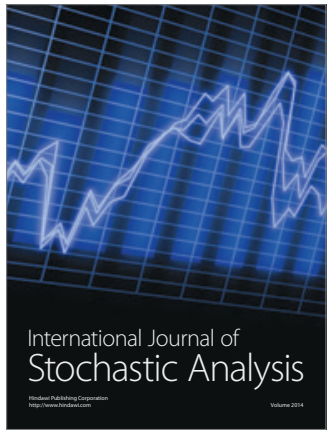

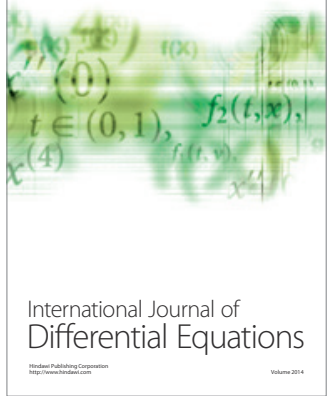
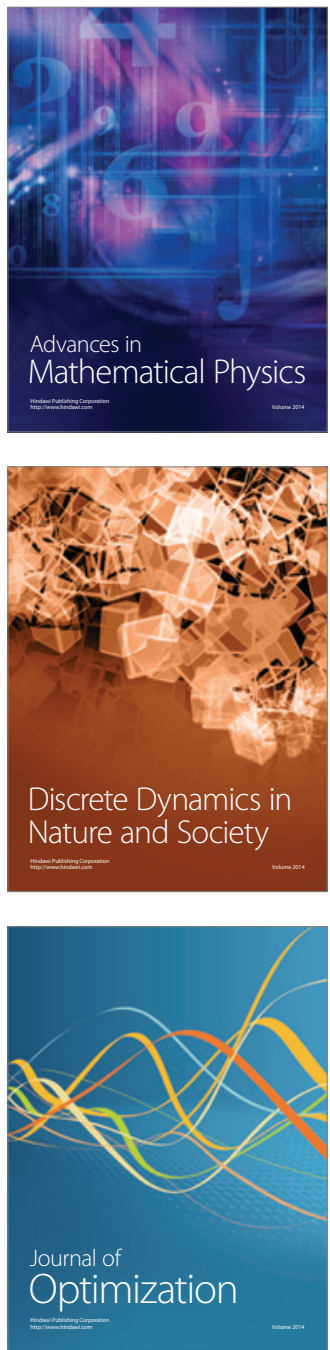